\title{
A Case of Large Anterior Paraclinoid Aneurysm with Intraoperative Premature Rupture
}

\author{
Shinya Ichimura ${ }^{1} \quad$ Makoto Inaba $^{1}$ Hiroshi Kagami $^{1}$ \\ ${ }^{1}$ Department of Neurosurgery, Saiseikai Yokohamashi Tobu Hospital, \\ Yokohama, Japan \\ J Neurol Surg Rep 2012;73:48-51. \\ Address for correspondence and reprint requests Shinya Ichimura, \\ M.D., Ph.D., Department of Neurosurgery, Saiseikai Yokohamashi Tobu \\ Hospital, 3-6-1 Shimosueyoshi, Tsurumi-ku Yokohama, Kanagawa, \\ Japan, 230-8765 (e-mail: shinya3917@gmail.com).
}

\begin{abstract}
Keywords

- anterior clinoid process

- anterior paraclinoid aneurysm

- premature rupture

- saccular aneurysm

- tentative clipping

\section{Introduction}

Aneurysms arising from the anterior wall of the internal carotid artery (ICA) are uncommon, reportedly comprising $0.3 \%$ to $1 \%$ of intracranial aneurysms or $0.9 \%$ to $6.5 \%$ of aneurysms of the ICA. ${ }^{1-5}$ Anterior paraclinoid aneurysms are defined as aneurysms arising from the anterolateral wall of the proximal ICA without any relationship to an arterial branch ${ }^{6}$; such aneurysms are immediately proximal to the anterior clinoid process. ${ }^{7-9}$ Removal of the anterior clinoid process is usually necessary for clipping of large or giant anterior paraclinoid aneurysms. We report here troubleshooting of intraoperative premature rupture of large anterior paraclinoid aneurysm, which was successfully clipped using incidental tentative clipping.
\end{abstract}

We report here troubleshooting of intraoperative premature rupture with large anterior paraclinoid aneurysm, which was successfully clipped. A 61-year-old woman with left nasal hemianopia was referred to our institute. Preoperative three-dimensional computed tomography angiography and a left internal carotid artery angiogram showed a large left anterior clinoid aneurysm adjacent to the anterior clinoid process. Aneurysm was ruptured prematurely and tentative clipping of the dome of the aneurysm was done incidentally to stop bleeding and to reduce the volume of the aneurysm. The anterior clinoid process and superior wall of the orbit were drilled out safely, since the tentative clipping had created sufficient space between the aneurysm and the anterior clinoid process to perform the procedure. The proximal neck was observed and tandem clipping was applied to the aneurysm. Intraoperative and postoperative angiography revealed complete disappearance of the aneurysm.

\section{Case Report}

\section{Clinical History}

A 61-year-old woman presented with a complaint of occasional headaches and was referred to our institution from another hospital with the diagnosis of unruptured left ICA aneurysm after a magnetic resonance imaging scan had revealed an abnormal flow void in the left parasellar region. A neurological examination disclosed left nasal hemianopia. On admission, a three-dimensional computed tomography angiogram (3D-CTA) ( - Fig. 1A) depicted a large aneurysm (20 $\mathrm{mm}$ in maximum diameter) adjacent to the anterior clinoid process and the superior wall of orbit projecting superomedially. The proximal side of the neck was observed under the face of the anterior clinoid process on the coronal view of the 3D-CTA ( - Fig. 1B). There was little space between the neck and the anterior clinoid process.

A left ICA angiogram revealed a large anterior paraclinoid aneurysm arising superiorly from the anterior surface of the ICA and separate from the ophthalmic artery ( - Fig. 2A). The view from the caudal side confirmed that the neck of the

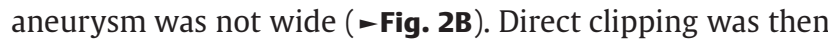
scheduled.

\section{Operation}

Prior to beginning the intracranial operation, the common, internal, and external carotid arteries were reserved at the common carotid artery bifurcation in the neck to secure them received

September 14, 2011

accepted after revision

February 3, 2012

published online

July 2, 2012
Copyright $\odot 2012$ by Thieme Medical Publishers, Inc., 333 Seventh Avenue, New York, NY 10001, USA. Tel: +1(212) 584-4662.
DOI http://dx.doi.org/ 10.1055/s-0032-1321504. ISSN 2193-6358. 


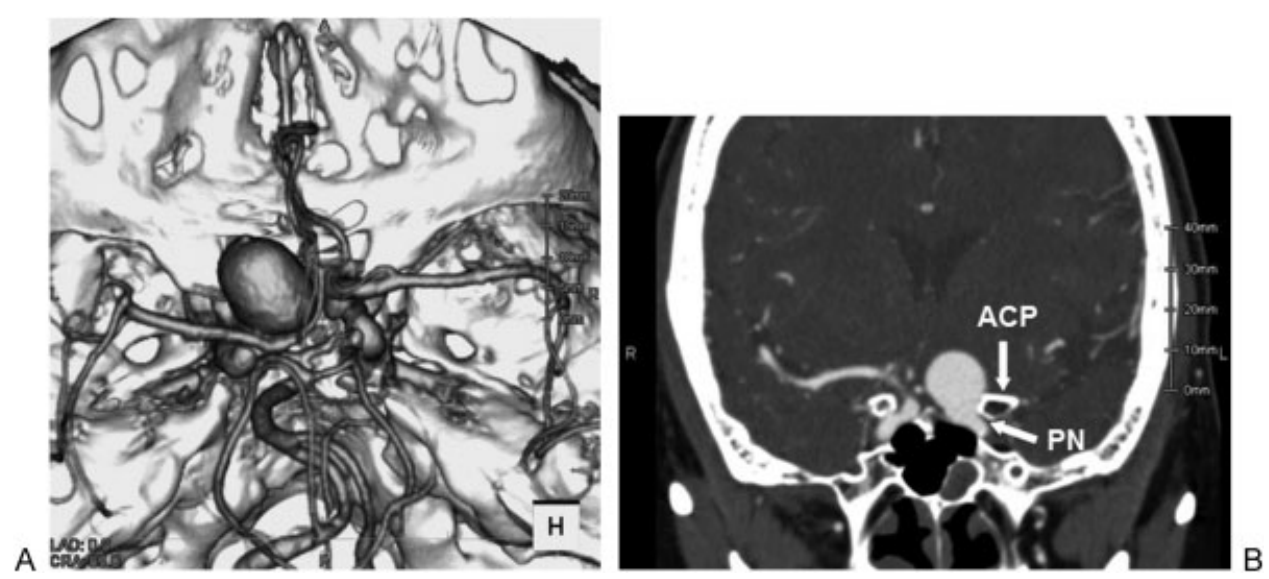

Figure 1 Preoperative three-dimensional computed tomography angiogram (3D-CTA) observed from above. (A) Large internal carotid artery adjacent to the anterior clinoid process and the anterior wall of the orbit. (B) 3D-CTA coronal view of the proximal side of the aneurysm neck under the anterior clinoid process with little space between the neck and the anterior clinoid process. ACP, anterior clinoid process; PN, proximal side of the aneurysm neck.
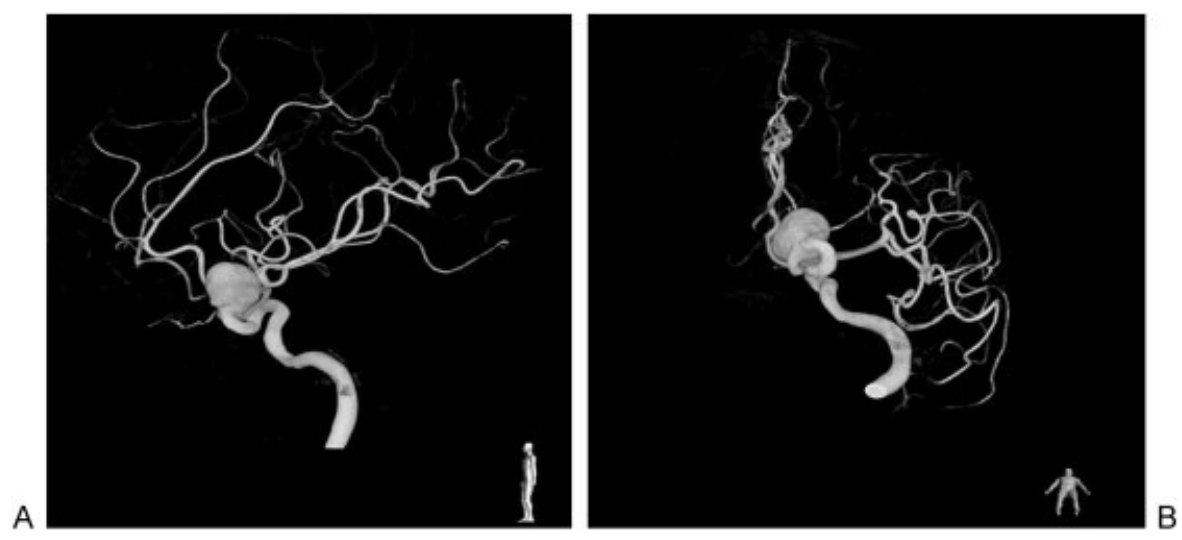

Figure 2 Preoperative left carotid artery angiograms. (A) Lateral view of a large anterior paraclinoid aneurysm, 20 mm in maximum diameter, at the left internal carotid artery projecting superomedially. The aneurysm was separate from the ophthalmic artery. (B) Caudal-side view showing that the neck of the aneurysm was not wide.

for proximal control. With the patient supine, a left frontotemporal craniotomy was performed to expose the ICA. After dissection of the Sylvian fissure and the carotid cistern, the anterior paraclinoid aneurysm was exposed on the anterior surface of the ICA. The aneurysm was adjacent to the anterior clinoid process and the superior wall of orbit ( $\mathbf{- F i g . ~ 3 A}$ ). The optic nerve was deviated superomedially, which was the reason for her left nasal hemianopia. The distal side of the neck of the aneurysm was observed at the proximal side of the anterior choroidal artery. Temporary clipping was done at the common, external carotid artery at the neck (proximal side of the aneurysm) and the ICA in the cranium (distal side of
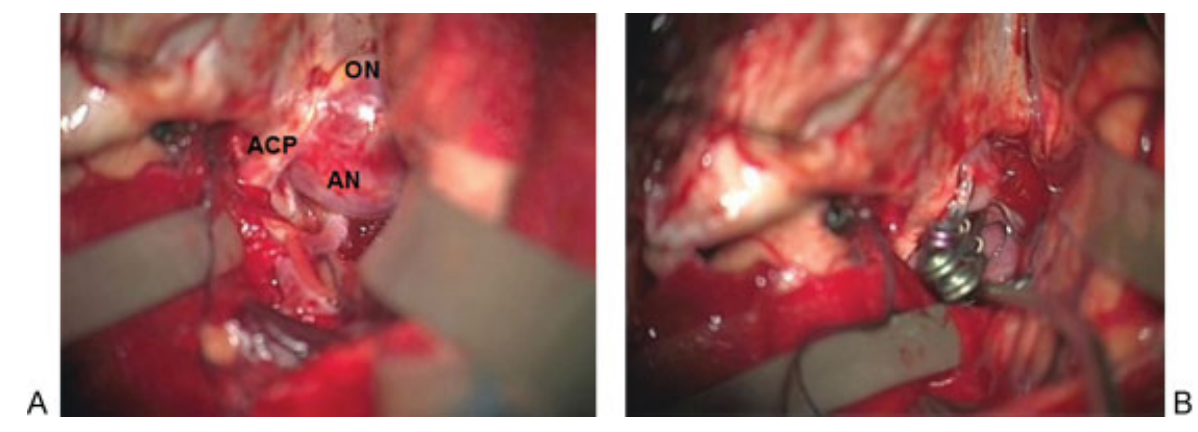

Figure 3 Intraoperative photographs: The aneurysm was adjacent to the anterior clinoid process and anterior orbital wall. The optic nerve was deviated superomedially (A). Tentative clipping was performed on the dome of the aneurysm (B). The proximal side of the neck of the aneurysm was exposed after removal of the anterior clinoid process and the anterolateral portion of the dural ring. The aneurysm was obliterated with tandem clipping. ACP, anterior clinoid process; AN, aneurysm; ON, optic nerve. 
the aneurysm). During the suction decompression cannulated from the ICA at the neck for 10 minutes, we tried to slide the permanent clip toward the proximal neck, but we couldn't because the proximal neck was invisible. The aneurysm was ruptured in this manipulation and tentative clipping was performed incidentally on the dome of the aneurysm parallel to the anterior clinoid process (-Fig. 3B). Three tentative clips were applied to stop bleeding. The volume of the aneurysm was reduced by tentative dome clipping. All temporary clippings were removed. The aneurysm was detached from the anterior clinoid process, the superior wall of the orbit, and the optic nerve. Tentative clipping created sufficient space between the aneurysm and the anterior clinoid process and to stop blood flow of the part of aneurysm near anterior clinoid process so that the anterior clinoid process could be safely drilled out epidurally. The anterior portion of the distal dural ring was cut, and the superior wall of the orbit was resected to mobilize the optic nerve. The proximal neck was observed under the face of the dissected anterior clinoid process. Angioplastic tandem clipping was applied to the aneurysm. After permanent clipping, the tentative clips were removed. Intraoperative angiography revealed that the aneurysm was angioplastically clipped parallel to the ICA. The wound was closed as usual.

\section{Postoperative Course}

The postoperative course was uneventful, but the patient's left nasal hemianopsia remained. Angiograms obtained after the surgery confirmed the complete disappearance of the aneurysm (-Fig. 4).

\section{Discussion}

The term "tentative clip" refers to a clip that enables the neck of an aneurysm to be temporarily clipped since it may also include a vessel that lowers the pressure of the aneurysm and so facilitates the next procedure. Tentative clipping is more appropriate when there is a vessel that is difficult to separate

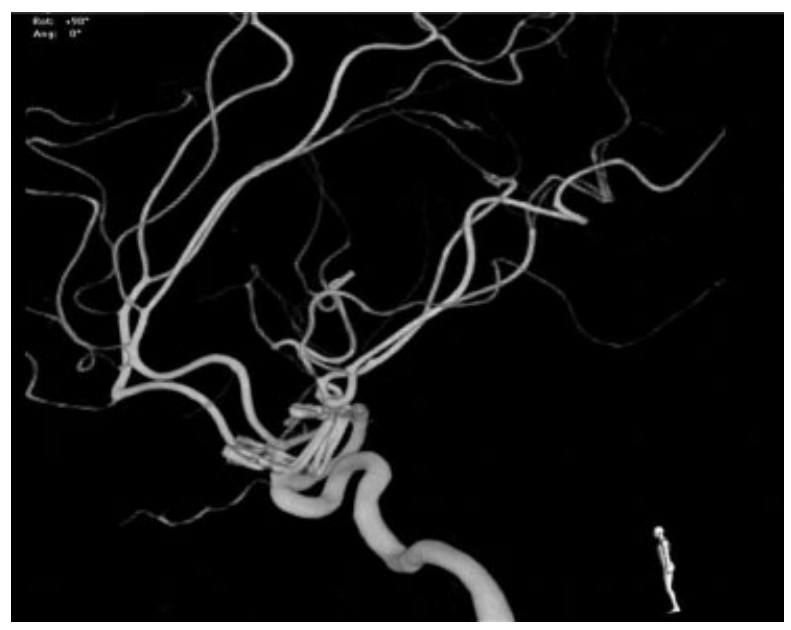

Figure 4 Postoperative angiography shows that the aneurysm was angioplastically clipped parallel to the internal carotid artery and complete disappearance of the aneurysm. from the back surface of a dome or for an aneurysm that includes a rupture point. A tentative clip has a lower tendency to cause brain ischemia than does a procedure in which a temporary clip includes the parent vessel, causing the blood flow of several branches to be temporarily interrupted. ${ }^{10-12}$

Anterior paraclinoid aneurysms are divided into a "blister type," with a blood blisterlike configuration and fragile walls and a "saccular type," with a saccular configuration and a relatively firm neck, typical of berry aneurysms. ${ }^{13-15}$ Saccular aneurysms can also occur at a nonbranching site along the anteromedial and anterior aspects of the supraclinoid segment of the ICA according to previous reports. ${ }^{13,15}$ Saccular
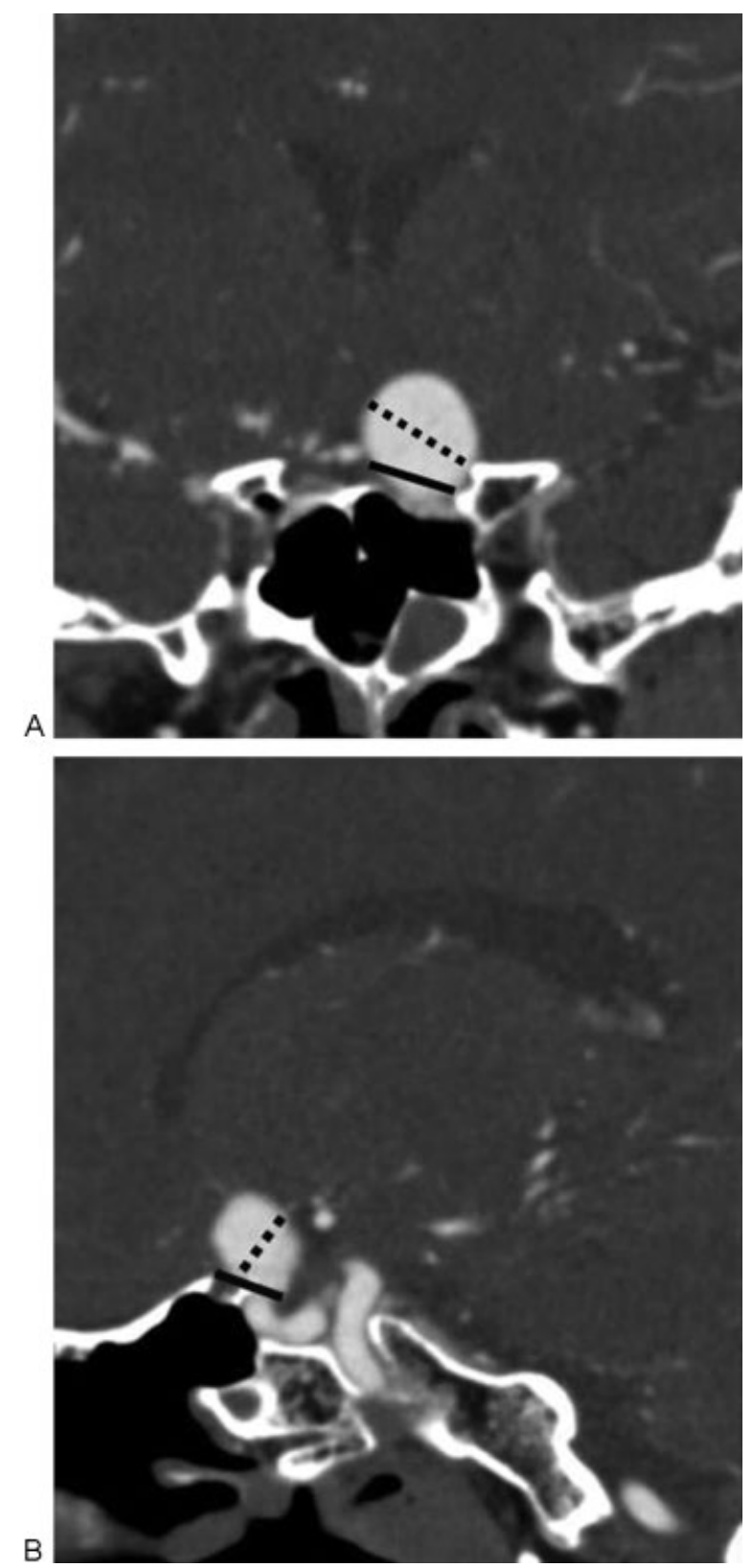

Figure 5 The closure line of tentative and permanent clipping. Tentative clipping does not reach the proximal neck of the aneurysm and is far from the distal neck of the aneurysm. (A) Coronal view. (B) Sagittal view. Dotted line, tentative clipping; straight line, permanent clipping. 
aneurysms have a firm wall and can be treated with neck clipping. ${ }^{16}$ Anterior paraclinoid aneurysms can be treated by clipping because these lesions are of the true berry type, are directed upwards, and have no relationship to any arterial branch. $^{7-9,14}$ The aneurysm is adjacent to the anterior clinoid process. Thus, removal of the anterior clinoid process is safer with direct visualization of the lesion after complete dissection of the Sylvian fissure and the carotid cistern and after confirmation of the anatomical relationship of the aneurysm to proximal control of the ICA. ${ }^{8}$

Drilling the anterior clinoid process to treat paraclinoid aneurysms can cause a catastrophic rupture of the aneurysm. $^{8}$ When aneurysms are located in this region, especially those projecting medially, removal of the anterior clinoid process, optic unroofing, and dissection of the dural ring are occasionally required to expose and obliterate the neck of the aneurysm. $^{17}$

In the present case, incidental tentative clipping provided a sufficient working space between the aneurysm and the anterior clinoid process, stopped the blood flow of the aneurysm adjacent to the anterior clinoid process, and reduced the volume of the aneurysm. - Fig. 5 shows the closure line of tentative and permanent clipping. Tentative clipping might not be an obstacle for permanent clipping because tentative clipping does not reach the proximal neck and is far from the distal neck of the aneurysm. To be sure, intradural clinoidectomy using the ultrasound aspirator to bony structure has been a well-known procedure for safe clinoidectomy in cases with anterior paraclinoid aneurysm; the tentative clipping parallel to the anterior clinoid process is an option for resecting the anterior clinoid process safely as troubleshooting of intraoperative premature rupture of large anterior paraclinoid aneurysm.

\section{References}

1 Abe M, Tabuchi K, Yokoyama H, Uchino A. Blood blisterlike aneurysms of the internal carotid artery. J Neurosurg 1998; 89:419-424

2 Kanamaru K, Ishida F, Taki W. Splitting and penetration of the optic nerve by an aneurysm arising from the anterior wall of internal carotid artery: case report. J Neurol Neurosurg Psychiatry 2001;71:525-527

3 Satoh A, Nakamura H, Odaki M, et al. High-risk aneurysm of the internal carotid artery: dorsal ICA aneurysms. Surg Cereb Stroke 1993;21:467-472

4 Tsuzuki N, Katoh H, Toyooka T, Uozumi Y, Shima K. Subarachnoid clot distribution in anterior wall saccular aneurysms of the internal carotid artery. J Clin Neurosci 2007;14:242-244

5 Yasargil MG. Microneurosurgery. Vol 2. Stuttgart: Thieme; 1984; pp 33-123.

6 Horiuchi T, Kusano Y, Yako T, Murata T, Kakizawa Y, Hongo K. Ruptured anterior paraclinoid aneurysms. Neurosurg Rev 2011; 34:49-55

7 Aldrich F. Anterior (dorsal) paraclinoid aneurysm: case report. Surg Neurol 1991;35:374-376

8 Kinouchi H, Mizoi K, Nagamine Y, et al. Anterior paraclinoid aneurysms. J Neurosurg 2002;96:1000-1005

9 Nishizaki T, Ikeda N, Kurokawa Y, Okamura T, Abiko S. Ruptured internal carotid artery anterior wall aneurysm identified during vasospasm: case report. Neurosurgery 2005;57:E811

10 Kato Y, Sano H, Okuma I, et al. Pitfalls in aneurysm surgery in acute stages. Neurol Res 1997;19:17-24

11 Sano H, Kato Y, Abe M, Kasama M, Kanno T. Utility and problems of temporary clips and tentative clips. Surg Cereb Stroke 1991;19: 595-597

12 Sano H, Kato Y, Kanno T. Treatment of giant intracranial aneurysms: a review of past cases and surgical techniques. Jpn J Neurosurg (Tokyo) 1992;1:339-347

13 Nagamine Y, Ogasawara K, Kinouchi H, Takahashi A, Yoshimoto T. Anterior wall aneurysms of internal carotid artery: "Chimame" type and "nonchimame" type. Surg Cereb Stroke 1997;25: 423-427

14 Nakagawa F, Kobayashi S, Takemae T, Sugita K. Aneurysms protruding from the dorsal wall of the internal carotid artery. J Neurosurg 1986;65:303-308

15 Ogawa A, Suzuki M, Ogasawara K. Aneurysms at nonbranching sites in the surpaclinoid portion of the internal carotid artery: internal carotid artery trunk aneurysms. Neurosurgery 2000;47: 578-583, discussion 583-586

16 Shimizu H, Matsumoto Y, Tominaga T. Non-saccular aneurysms of the supraclinoid internal carotid artery trunk causing subarachnoid hemorrhage: acute surgical treatments and review of literatures. Neurosurg Rev 2010;33:205-216

17 Hongo K, Watanabe N, Matsushima N, Kobayashi S. Contralateral pterional approach to a giant internal carotid-ophthalmic artery aneruysm: technical case report. Neurosurgery 2001;48:955-957, discussion 957-959 